\title{
Murine Monoclonal Antibody 225
}

National Cancer Institute

\section{Source}

National Cancer Institute. Murine Monoclonal Antibody 225. NCI Thesaurus. Code C38116.

A mouse monoclonal antibody that was raised against human epidermal growth factor (EGF) receptor. Antibody 225 competes with EGF or transforming growth factor-alpha (T GF alpha) for receptor binding and thereby prevents lig and-induced receptor tyrosine kinase activation resulting in the inhibition of proliferation of human cancer cells that are stimulated by the T GFalpha/EGF receptor autocrine loop. 